\title{
Vertically aligned nanostructured gold microtube assisted by polymer template with combination of wet phase inversion and $\mathrm{Cu}$ grid mask
}

\begin{abstract}
Soohyun $\mathrm{Kim}^{1}$ \& Keon-Soo Jang ${ }^{2 \bowtie}$
Tubular architecture has been extensively exploited in diverse applications such as solar cells and sensors. However, the synthesis of microtubes with high aspect ratio using polymer templates has been rarely reported. In this study, we designed a facile avenue for the synthesis of well-aligned Au nanoparticle-agglomerate microtubes with an aspect ratio of $\sim 30$ using a hollow polyetherimide (PEI) template. The combination of wet phase inversion and use of a $\mathrm{Cu}$ grid mask enabled straightforward production of a hollow PEI template with vertically aligned tubular architecture. During wet-phase inversion, exchange between a solvent ( $N$-methyl-2-pyrrolidone) and a non-solvent (water) occurred at the corners of the square mask cells rather than along their side, thereby producing pores at the corners due to geometrical and entropic factors. The hollow microtubes were comprised of agglomerated Au nanoparticles that coated the inner surfaces of the pores during an electroless plating process performed after wet-phase inversion. This finding is applicable to diverse applications such as sensors and catalysis.
\end{abstract}

A recent interest in hollow microtubular architecture has surfaced over the past two decades due to the unique physical and chemical properties, such as unprecedented aspect ratio, high specific surface area, and excellent electrical conductivity ${ }^{1-6}$. The tubular microstructures have been exploited in a myriad of applications such as optoelectronics, actuators, sensing, 3D cell microreactors, and catalysis ${ }^{7-18}$. Various approaches have been introduced to fabricate metallic and inorganic microtubes. For instance, $\mathrm{ZnO}$ microtubes were manufactured on Si substrates by chemical vapor deposition ${ }^{19}$. Metal precursors were mixed with polyacrylonitrile (PAN) in ethylene glycol and then the electrospun PAN composite was degraded to produce metal microtubes ${ }^{20}$. 2D hexagonal pore arrays were fabricated by the combination of macroporous $\mathrm{Si}$ template and deep reactive ion etching, followed by chemical solution method to make ferroelectric microbutes ${ }^{21}$. Rolling-up process achieved the fabrication of diverse metal microtubes ${ }^{7,16,17,22}$.

Among various microtubes, gold $(\mathrm{Au})$ microtubes are particularly useful in a wide range of applications as a possible use in optics, information storage, optoelectronics, separation, sensing, actuators, microelectromechanical devices, and bio sensing devices due to their excellent electrochemical stability, biocompatibility, insusceptibility to oxidation, chemical inertness, and catalysis ${ }^{1,3-6,23-29}$. Au plating can be achieved by electroless plating, electrolytic plating, and e-beam evaporation/lithography ${ }^{30-34}$. Similar to other metal microtubes, gold microtubes have also been fabricated by these various templating/plating methods and special designs. In particular, Au nanoparticles are especially useful to further improve the specific surface area of microsized-structures. For example, Au nanoparticles have been employed as microtube building blocks in biosensors, electronic nanodevices, and applications that employ surface-enhanced Raman scattering, and kapok fiber composite ${ }^{3,35-37}$.

Among various templating methods, the use of a polyetherimide (PEI) template for constructing cylindrical porous structures is promising ${ }^{38}$, and has garnered considerable attention due to its facile fabrication ${ }^{39-41}$. The PEI polymer renders self-growing cylindrical porous structures through a wet phase inversion process ${ }^{39,41}$. However, the random distribution of cylindrical pores in the PEI template is a critical problem. In the present study, we produced a hollow polymer template by a facile process that combined the use of a Cu grid mask with

${ }^{1}$ LG Chem, Magokjungang 10-ro, Gangseo-gu, Seoul 07796, Republic of Korea. ${ }^{2}$ Department of Polymer Engineering, School of Chemical and Materials Engineering, The University of Suwon, Hwaseong, Gyeonggi-do 18323, Republic of Korea. ${ }^{\bowtie}$ email: ksjang@suwon.ac.kr 
wet phase inversion to achieve a $1 \mathrm{D}$ hierarchically aligned Au microtubular structure. This combination led to high aspect ratio over a large area. Owing to its simplicity and versatility, the approach presented herein allows promising strategy for the functionalization of tubular structures.

\section{Experimental}

Materials. Analytical grade mercaptosuccinic acid powder (TCI, Japan), 1-hexadecanethiol (Sigma-Aldrich Co., St. Louis, MO, USA), tin(II) chloride $\left(\mathrm{SnCl}_{2}\right.$, Mallinckrodt Baker Inc., Phillipsburg, NJ, USA), trifluoroacetic acid (Sigma-Aldrich) gamma-butyrolactone (GBL, Acros Organics Co. Belgium), methanol (BNOChem, South Korea), PEI (Sigma-Aldrich), nitric acid ( $\mathrm{HNO}_{3}$, Sigma-Aldrich), formaldehyde (HCHO, Sigma-Aldrich), $\mathrm{N}$-methyl-2-pyrrolidone (NMP, Sigma-Aldrich), silver nitrate $\left(\mathrm{AgNO}_{3}\right.$, Sigma-Aldrich), sodium sulfite $\left(\mathrm{Na}_{2} \mathrm{SO}_{3}\right.$, Sigma-Aldrich), and sodium bicarbonate $\left(\mathrm{NaHCO}_{3}\right.$, Junsei Chemical Co. Japan) were used as received. A 2000mesh square $\mathrm{Cu}$ grid was purchased from Gilder Grids (UK) and used as a mask. The walls of the grid were $5 \mu \mathrm{m}$ thick, and the openings were $7.5 \mu \mathrm{m} \times 7.5 \mu \mathrm{m}$ in size. Oromerse gold electroless plating solution was purchased from Technic Inc. (Cranston, RI, USA).

Preparation of masking with thiol treatment. For treatment of masking, $12 \mathrm{mg}$ of mercaptosuccinic acid and $20 \mathrm{mg}$ of 1-hexadecanethiol solution were dissolved in $40 \mathrm{~mL}$ of ethanol for hydrophilic and hydrophobic treatment of the $\mathrm{Cu}$ grid mask, respectively (Fig. S1). Subsequently, the Cu grid mask was immersed into the solution for $2 \mathrm{~h}$. After treatment, the treated mask was carefully retrieved.

Fabrication of aligned PEI template. A $10 \mathrm{wt} \%$ solution of PEI for fabrication of finger-like microstructures was prepared by dissolving PEI in a solvent mixture (NMP:GBL $=6: 4$ vol $\%$ ). The mixture containing NMP and viscous GBL was used as a solvent for wet phase inversion, with GBL controlling the viscosity. The solution was stirred at $70{ }^{\circ} \mathrm{C}$ for $5 \mathrm{~h}$. The solution was then cast on a glass slide substrate by doctor-blading. Tape was utilized to control the depth of the solution. After casting at room temperature, a PEI membrane was obtained, onto which the thiol-treated mask was immediately placed. Then, it was immersed in a coagulation medium with water. The solution was inversed from the liquid phase to the solid phase over the course of $4 \mathrm{~h}$. Thereafter, the membrane was immersed in methanol for 1 day, followed by thermal treatment at $230^{\circ} \mathrm{C}$ for $15 \mathrm{~h}$ to attain a smooth membrane surface.

Preparation of Au structure in template by electroless plating. Electroless plating involves oxidation and reduction (Fig. S2). In the first step of this process, the membrane was wetted with methanol and then immersed in a 1:1 (v/v) methanol-water solution containing $0.025 \mathrm{M} \mathrm{SnCl}_{2}$ and $0.07 \mathrm{M}^{2}$ trifluoroacetic acid for $45 \mathrm{~min}$. The membrane was rinsed with methanol. After sensitization with $\mathrm{Sn}^{2+}$, the membrane was activated in ammoniacal $\mathrm{AgNO}_{3}$ solution $(0.029 \mathrm{M})$ for $5 \mathrm{~min}$. The membrane was then washed with methanol. For Au plating, the membrane was immersed in an Au plating bath ( $\mathrm{pH} 10)$ consisting of $7.9 \times 10^{-5} \mathrm{M} \mathrm{Na}_{3} \mathrm{Au}\left(\mathrm{SO}_{3}\right)_{2}, 0.127 \mathrm{M}$ $\mathrm{Na}_{2} \mathrm{SO}_{3}$, and $0.625 \mathrm{M}$ formaldehyde at $5{ }^{\circ} \mathrm{C}$. The membrane was cleaned in dilute $\mathrm{HNO}_{3}$ solution for $12 \mathrm{~h}$ and immersed in methanol. Then, the membrane was immersed in NMP for 1 day to remove the PEI template, and the remaining array was rinsed with deionized-water.

Characterization. Scanning electron microscopy (SEM, APREO, FEI Co., Hillsboro, OR, USA) with an energy dispersive spectroscopy (EDS) at the Center for Advanced Materials Analysis was performed to investigate the morphologies of the template and gold arrays. The samples on the carbon surfaces were sputter-coated with Pt/Pd. For cross-sectional SEM images, the polymer template was cryo-fractured in liquid nitrogen whereas the aligned Au microtubes were easily detached with tweezers.

Confocal microscopy (FluoView FV1000, Olympus Corp., Japan) was carried out for two (x, y)-dimensional high-resolution imaging of histocultured whiskers with laser excitation at $470 \mathrm{~nm}$ to observe the morphology of the Cu mask.

\section{Results and discussion}

Highly ordered Au arrays were fabricated by pairing the PEI templating with electroless Au plating, as detailed in Fig. 1. A continuous and uniform porous PEI membrane film as a sacrificial template with an ordered 1D structure was prepared via phase inversion. This phase inversion occurred via the exchange process between a solvent (NMP) and a nonsolvent (DI-water) in a coagulation bath. Phase inversion resulted from transformation of an initially thermodynamically stable polymer solution to an unstable state via the solvent/nonsolvent exchange during coagulation. The resulting membrane structure was composed of a uniform cylindrical pore (honeycomb) structure with a pore diameter range of 3-5 $\mu \mathrm{m}$. However, the cylindrical porous polymer film fabricated by wet phase inversion generally exhibit a random distribution of pores ${ }^{42}$. In this work, homogeneously aligned structures were obtained by placing a copper mask (Fig. 1a) onto the PEI solution prior to immersion; this process induced tunable and aligned nucleation sites for pore generation on the PEI template film. After the wet phase inversion of the copper mask/PEI solution sample, the mask was easily detached with tweezers. Then, a thin coat of $\mathrm{Au}$ was deposited onto the remaining porous template by electroless Au plating. The sacrificial polymer template was removed by NMP. Through this process, well-aligned hollow Au rods were successfully manufactured as shown in Fig. 2.

Visual observation of polymer templates has been routinely carried out through SEM and confocal microscopy. Figure $2 \mathrm{~b}$ shows a top view of the well-ordered patterns on the PEI film morphology after detachment of the copper mask in the coagulation bath. The regular polymer prominence and depression morphology by mask 

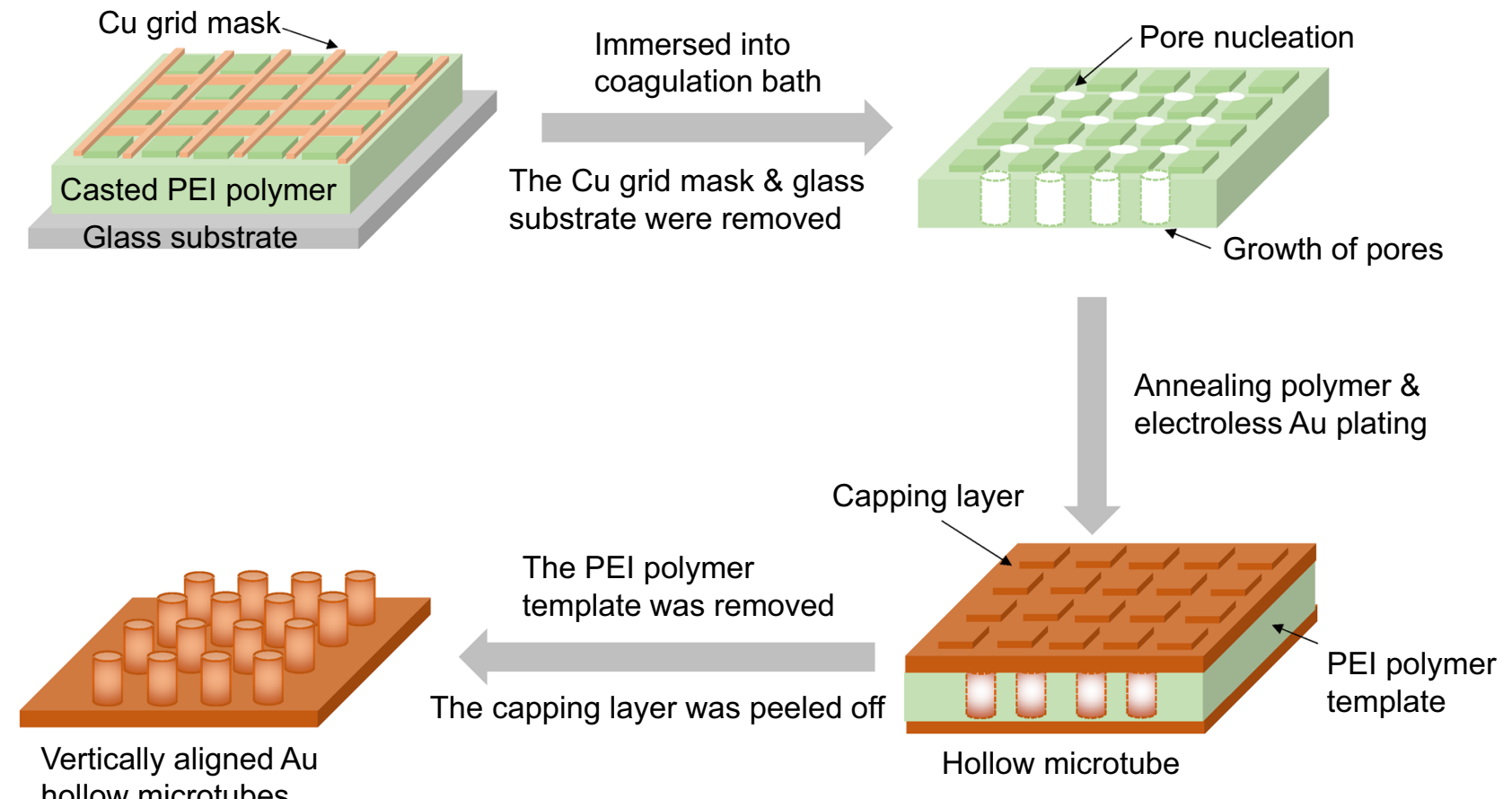

Figure 1. Vertically aligned gold microtube fabrication process.
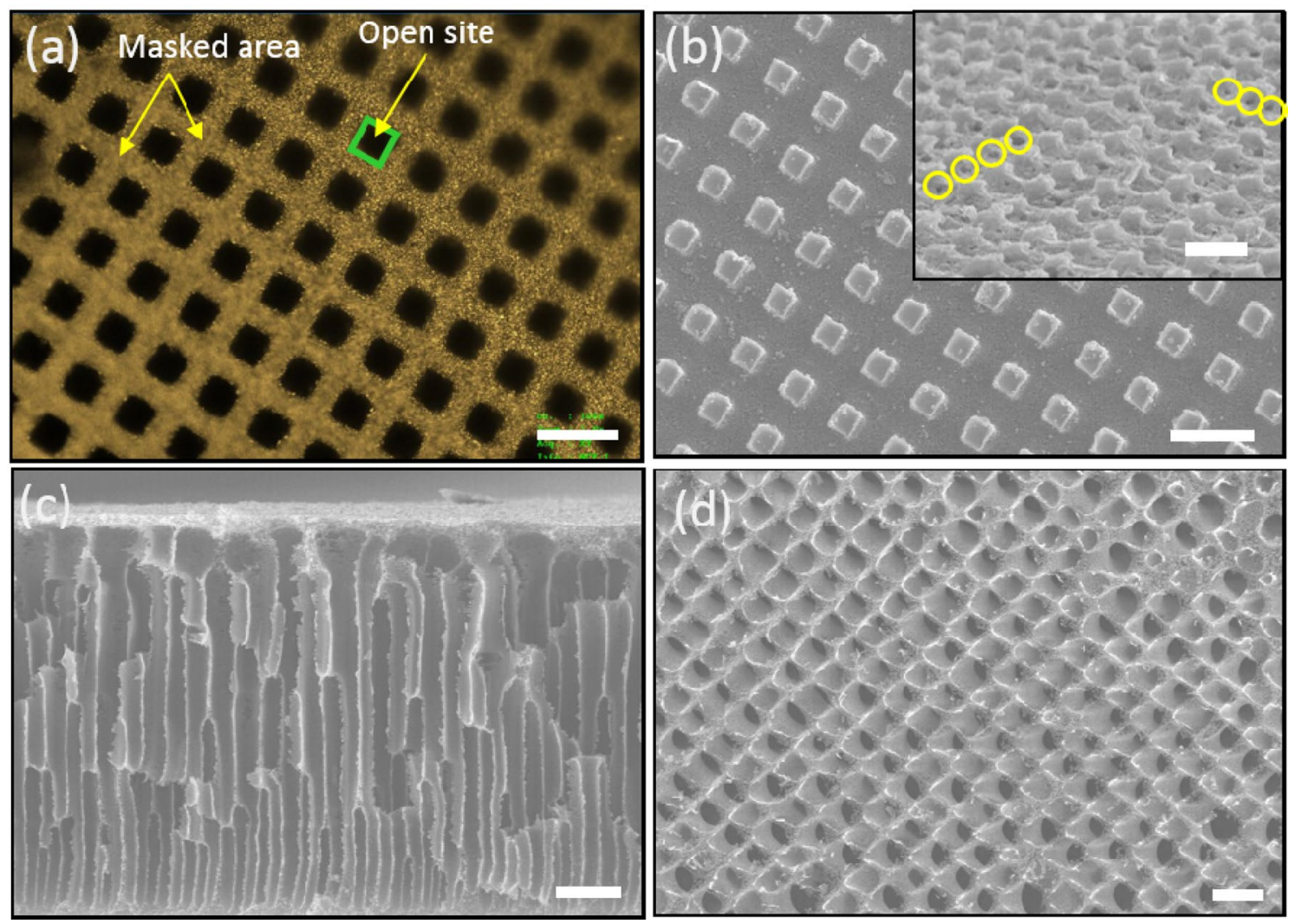

Figure 2. Confocal images of Cu mask (a) and SEM images of PEI polymer template (b-d): (b) top view of PEI template (inset: tilt image of PEI template, with yellow circles indicating the nucleation sites), (c) cross section of PEI template, and (d) highly aligned interior of PEI template after 5 min of oxygen plasma etching. The scale bar is $15 \mu \mathrm{m}$. 

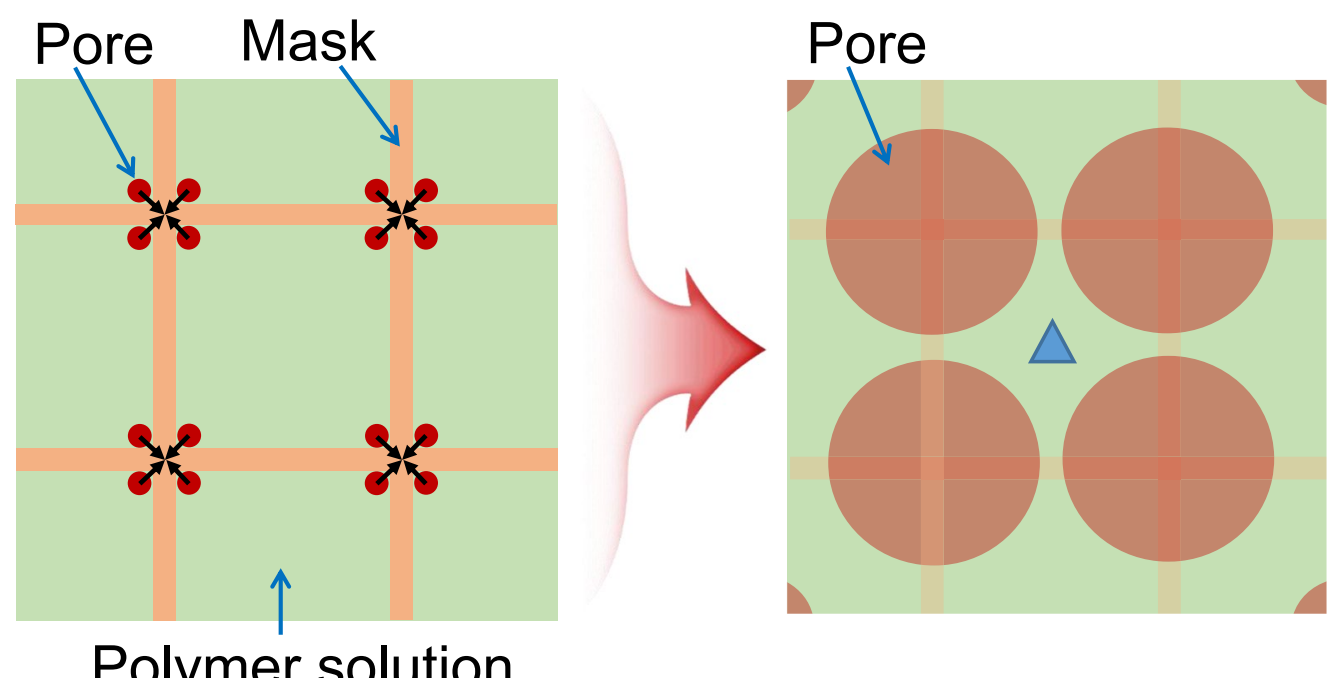

\section{Polymer solution}

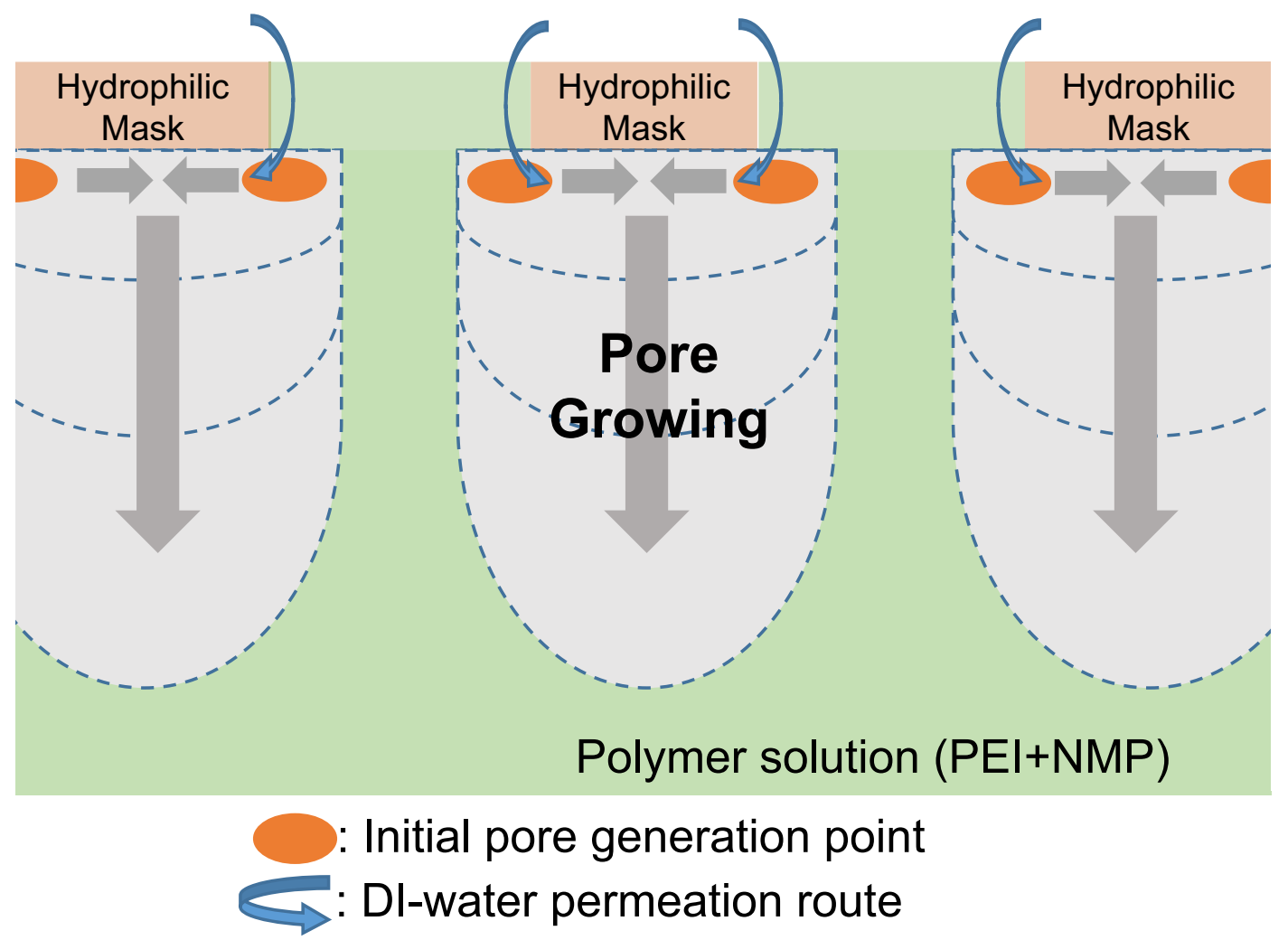

Figure 3. Wet phase inversion process: Initial pore generation at edges of masks and pore growth underneath intersection of mask lines.

were observed on the top of PEI polymer film. The tilt inset of Fig. $2 \mathrm{~b}$ shows the prominent/depressed structure with a nucleation pore marked by yellow circles. The open site (prominent site) underwent the phase inversion between the solvent and the non-solvent. The morphology was confirmed with $2 \mathrm{D}$ images obtained by confocal microscopy (Fig. S3). A well-aligned porous structure in the polymer template with copper masking (Fig. S3a-c) and an irregular pore structure without copper masking (Fig. S3d-f) via wet phase inversion were observed. The regularly spaced pore nuclei were observed over a large area on the top of polymer film. The pore nucleation sites were thought to be located at the edge (corner) of the square mask cells due to a geometric effect. The probability of wet phase inversion at the edges of each mask cell was higher than that along the sides of the mask cells.

Figure $2 \mathrm{c}$ shows a cross section of the cylindrical PEI templates with no pore distortion owing to the $\mathrm{Cu}$ mask. Figure S4 shows the free-standing structure of the Au micro-rods without distortion or damages following dissolution of the polymer template by solvent or electroless Au plating. The large specific surface area 

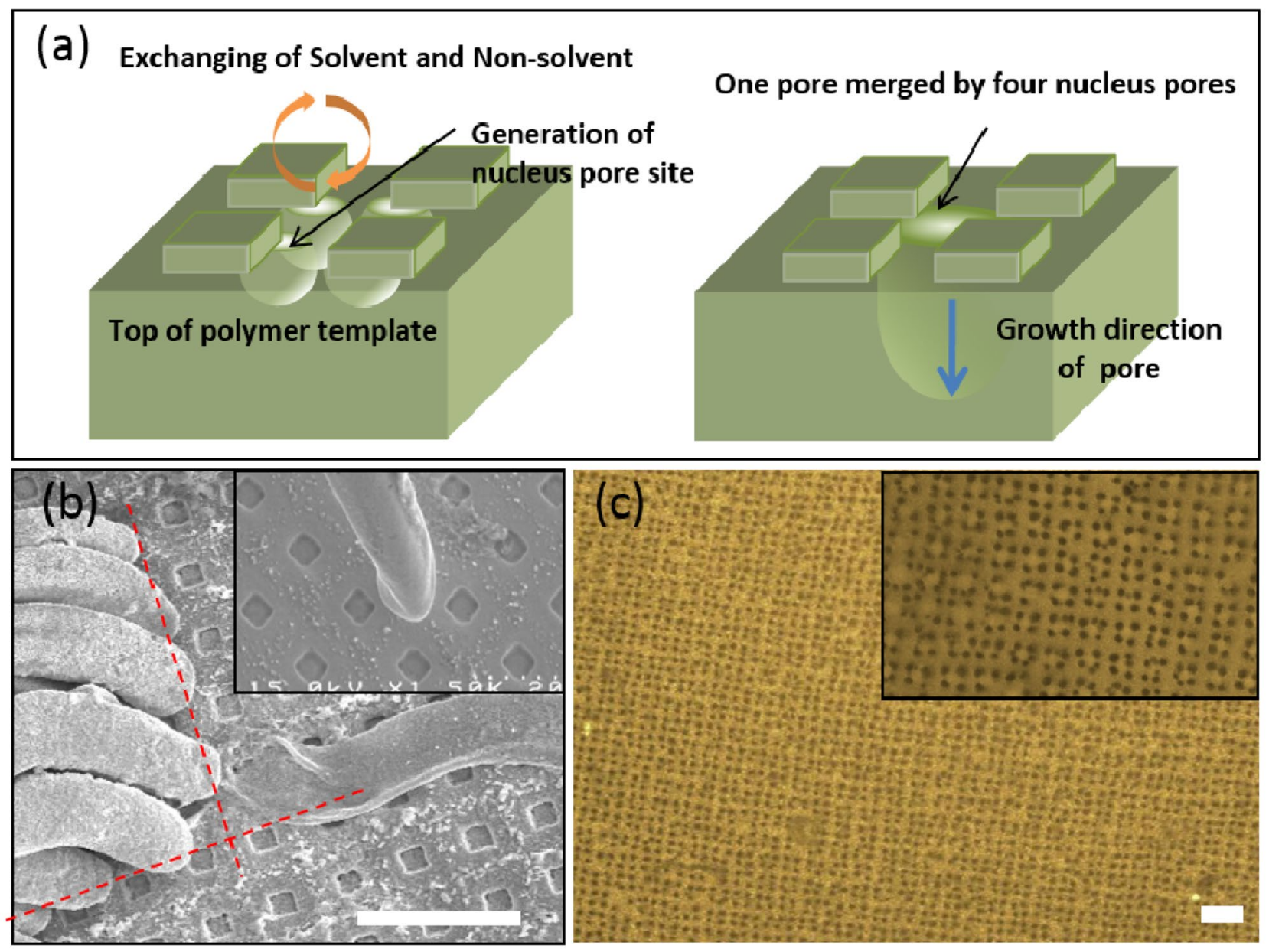

Figure 4. Schematic of mechanism and SEM images (scale bar is $20 \mu \mathrm{m})$ : (a) pore generation mechanism, (b) a bundle of Au-capped rods (inset: single Au rod), (c): confocal image of nucleation pores on PEI membrane (inset: magnified images).

could facilitate the application of diverse catalysts and electrochemical devices. To further probe regularity of the aligned cylindrical structure from top to bottom, oxygen plasma etching was performed. Figure $2 \mathrm{~d}$ shows the interior morphology of the cylindrical PEI template after $300 \mathrm{~s}$ of oxygen plasma etching over the top of the template. This indicates a perfectly aligned interior pore structure. Figure S5 exhibits the SEM images of the interior structure of the polymer template as a function of etching time prior to Au electroless plating. Some top prominent structures remained after $30 \mathrm{~s}$ of etching (Fig. S5a). At $60 \mathrm{~s}$ of etching time of the oxygen plasma, pores were formed in the top layer of polymer template between the prominences (Fig. S5b). Finally, the well-aligned porous arrays were obtained at $120 \mathrm{~s}$ of etching time (Fig. S5d). Figures $2 \mathrm{a}-\mathrm{d}$ demonstrate that the sacrificial template allowed formation of the well-aligned interior structure.

The porous structure was generated underneath intersection of mask lines via initial pore generation at edges of masks as shown in Fig. 3. The initially produced small pores at edges were coalesced nearest to each pore, thereby leading to large porous structure underneath the intersection of mask lines. However, it might have been located at the center of polymer solution (blue triangle) in Fig. 3, if the mask lines were substantially thick due to closest distance between initial pores.

The mechanism of pore nucleus generation on the PEI template via phase inversion is illustrated in Figs. 3 and $4 \mathrm{a}$. Prior to phase inversion, the pore nuclei emerged at the edges (corners) of the square cells of the mask grid on the upper surface of the PEI polymer film (Fig. 4). This phenomenon facilitated inversion at the corners of the square cells, but not near their center. Upon addition of the solvent, the polymer-phase with a solvent underwent a transition from a homogeneous mixture to a polymer-rich and polymer-poor phases. Pores formed in the polymer-poor phase and formed preferentially at the cell corners because the geometric and entropic conditions there were more favorable for pore formation than they were along the sides of the cells. The pore nuclei generated at the corners of four mask cells merged to form a larger pore $(3 \mu \mathrm{m}$ in diameter), which spanned the full vertical length of the PEI film. Au rods were generated via electroless plating of the inner pore walls, which corresponded to the open areas of the $\mathrm{Cu}$ mask grid.

The PEI template was annealed at $230^{\circ} \mathrm{C}$ for $15 \mathrm{~h}$ to remove some porous sites of the PEI wall, thereby achieving a smooth wall of interior template for the hollow Au rod ${ }^{43}$. The prominence and depression structures were obtained by Au plating, as shown in Fig. S4. The SEM images indicate that the polymer template remained 


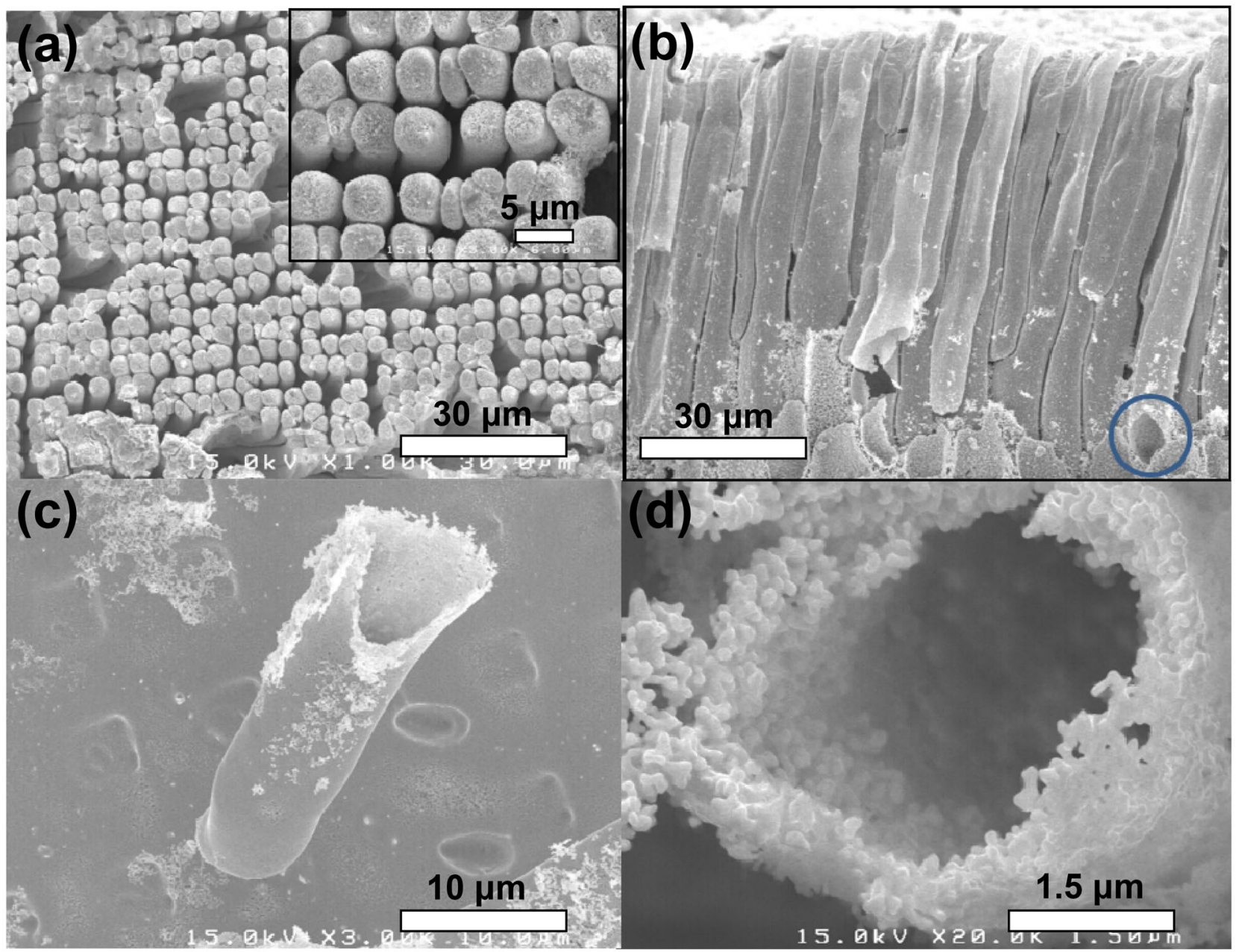

Figure 5. SEM images of gold microtubes. Top view (a) and cross sectional view (b) of aligned Au microtube. Single Au microtube (c,d).

undamaged without distortion during annealing and electroless Au plating. As shown in Fig. 5b, a thin Au capping layer grew on the rod during electroless Au plating. After dissolving the polymer template in the solvent (NMP), well-aligned hollow Au rod arrays with a diameter of 3-5 $\mathrm{m}$ and height of $80-90 \mu \mathrm{m}$ (Fig. 5b) were obtained by carefully peeling the capping layer on the top of Au microtubes (Fig. S6). The Au capping layer was easily removed with tweezers due to the empty space (originally the polymer template) between the Au capping layer and $\mathrm{Au}$ rod arrays. The aspect ratio of 20-30 was analogous to the dimensions of the template. The rods were rarely collapsed by a capillary force. Gold nanoparticles (submicron) were highly packed and assembled into a hollow tubular morphology with a micrometer scale of 3-5 $\mu \mathrm{m}$ in Figs. 5c,d. Small metal nanoparticles tend to aggregate to minimize their surface area. In this study, the rod structures were chiefly composed of $\mathrm{Au}$ (Fig. S7), following the removal of the template.

\section{Conclusion}

The facile fabrication of well-aligned hollow Au rods was achieved using a PEI polymer template and electroless $\mathrm{Au}$ deposition. A cylindrical porous polymer template was simply generated via phase inversion. This procedure is readily amenable to scaling up and will allow for the exploration of electronic, optical, chemical and physical properties for practical applications. The results of this work may serve as a reference for nanotube fabrication via lithography using a nano-patterned $\mathrm{Cu}$ mask. These nanotubes are useful in nanoscale applications, such as nanoelectronics, sensors, and catalysts.

Received: 15 July 2020; Accepted: 15 September 2020

Published online: 02 October 2020

\section{References}

1. Paczesny, J. et al. Hollow microtubes made of carbon, boron and gold: novel semiconducting nanocomposite material for applications in electrochemistry and temperature sensing. RSC Adv. 5, 64083-64090 (2015). 
2. Monk, D. J. \& Walt, D. R. Fabrication of gold microtubes and microwires in high aspect ratio capillary arrays. J. Am. Chem. Soc. 126, 11416-11417 (2004).

3. Wang, T., Zheng, R., Hu, X., Zhang, L. \& Dong, S. Templated assembly of gold nanoparticles into microscale tubules and their application in surface-enhanced raman scattering. J. Phys. Chem. B 110, 14179-14185 (2006).

4. M. Fontes, A. et al. Bio-inspired gold microtubes based on the morphology of filamentous fungi. Biomater. Sci.2, 956-960 (2014).

5. Pal, U. et al. Nanoparticle-assembled gold microtubes built on fungi templates for SERS-based molecular sensing. ACS Appl. Nano Mater. 2, 2533-2541 (2019).

6. Bianchini, C., Zane, D. \& Curulli, A. Gold microtubes assembling architecture for an impedimetric glucose biosensing system. Sensors Actuators B Chem. 220, 734-742 (2015).

7. Huang, G. et al. Rolled-up optical microcavities with subwavelength wall thicknesses for enhanced liquid sensing applications. ACS Nano 4, 3123-3130 (2010).

8. Yang, G., White, I. M. \& Fan, X. An opto-fluidic ring resonator biosensor for the detection of organophosphorus pesticides. Sensors Actuators B Chem. 133, 105-112 (2008).

9. White, I. M., Oveys, H., Fan, X., Smith, T. L. \& Zhang, J. Integrated multiplexed biosensors based on liquid core optical ring resonators and antiresonant reflecting optical waveguides. Appl. Phys. Lett. 89, 191106 (2006).

10. Sanchez, S., Solovev, A. A., Harazim, S. M. \& Schmidt, O. G. Microbots swimming in the flowing streams of microfluidic channels. J. Am. Chem. Soc. 133, 701-703 (2011).

11. Sanchez, S., Solovev, A. A., Schulze, S. \& Schmidt, O. G. Controlled manipulation of multiple cells using catalytic microbots. Chem. Commun. 47, 698-700 (2010).

12. Solovev, A. A., Sanchez, S., Pumera, M., Mei, Y. F. \& Schmidt, O. G. Magnetic control of tubular catalytic microbots for the transport, assembly, and delivery of micro-objects. Adv. Funct. Mater. 20, 2430-2435 (2010).

13. Sanchez, S., Solovev, A. A., Mei, Y. \& Schmidt, O. G. Dynamics of biocatalytic microengines mediated by variable friction control. J. Am. Chem. Soc. 132, 13144-13145 (2010).

14. Sanchez, S., Ananth, A. N., Fomin, V. M., Viehrig, M. \& Schmidt, O. G. Superfast motion of catalytic microjet engines at physiological temperature. J. Am. Chem. Soc. 133, 14860-14863 (2011).

15. Balasubramanian, S. et al. Micromachine-enabled capture and isolation of cancer cells in complex media. Angew. Chem. 123, 4247-4250 (2011).

16. Mönch, I. et al. Rolled-up magnetic sensor: nanomembrane architecture for in-flow detection of magnetic objects. ACS Nano 5, 7436-7442 (2011)

17. Huang, G., Mei, Y., Thurmer, D. J., Coric, E. \& Schmidt, O. G. Rolled-up transparent microtubes as two-dimensionally confined culture scaffolds of individual yeast cells. Lab Chip 9, 263-268 (2009).

18. Yu, M. et al. Semiconductor nanomembrane tubes: three-dimensional confinement for controlled neurite outgrowth. ACS Nano 5, 2447-2457 (2011).

19. Sun, T. \& Qiu, J. Fabrication of ZnO microtube arrays via vapor phase growth. Mater. Lett. 62, 1528-1531 (2008).

20. Zhang, M. et al. Bi2MoO6 microtubes: controlled fabrication by using electrospun polyacrylonitrile microfibers as template and their enhanced visible light photocatalytic activity. J. Hazard. Mater. 225-226, 155-163 (2012).

21. Bharadwaja, S. S. N. et al. Fabrication of high aspect ratio ferroelectric microtubes by vacuum infiltration using macroporous silicon templates. J. Am. Ceram. Soc. 89, 2695-2701 (2006).

22. Harazim, S. M., Xi, W., Schmidt, C. K., Sanchez, S. \& Schmidt, O. G. Fabrication and applications of large arrays of multifunctional rolled-up $\mathrm{SiO} / \mathrm{SiO}_{2}$ microtubes. J. Mater. Chem. 22, 2878-2884 (2012).

23. Sanchez-Castillo, M. A., Couto, C., Kim, W. B. \& Dumesic, J. A. Gold-nanotube membranes for the oxidation of CO at gas-water interfaces. Angew. Chem. 116, 1160-1162 (2004).

24. Taton, T. A., Mirkin, C. A. \& Letsinger, R. L. Scanometric DNA array detection with nanoparticle probes. Science 289, 1757-1760 (2000).

25. Nicewarner-Peña, S. R. et al. Submicrometer metallic barcodes. Science 294, 137-141 (2001).

26. Jirage, K. B., Hulteen, J. C. \& Martin, C. R. Nanotubule-based molecular-filtration membranes. Science 278, 655-658 (1997).

27. Chen, S. \& Yang, Y. Magnetoelectrochemistry of gold nanoparticle quantized capacitance charging. J. Am. Chem. Soc. 124, 52805281 (2002).

28. Maier, S. A. et al. Plasmonics-a route to nanoscale optical devices. Adv. Mater. 13, 1501-1505 (2001).

29. Sun, S., Murray, C. B., Weller, D., Folks, L. \& Moser, A. Monodisperse FePt nanoparticles and ferromagnetic FePt nanocrystal superlattices. Science 287, 1989-1992 (2000).

30. Kobayashi, Y., Tadaki, Y., Nagao, D. \& Konno, M. Deposition of gold nanoparticles on silica spheres by electroless metal plating technique. J. Colloid Interface Sci. 283, 601-604 (2005).

31. Jang, G.-G. \& Roper, D. K. Continuous flow electroless plating enhances optical features of Au films and nanoparticles. J. Phys. Chem. C 113, 19228-19236 (2009).

32. Li, B., Li, N., Luo, G. \& Tian, D. Acceleration effect of $\mathrm{Na}_{2} \mathrm{~S}_{2} \mathrm{O}_{3}$ on the immersion gold plating on Ni-P surface from a sulfite based electrolyte. Surf. Coat. Technol. 302, 202-207 (2016).

33. Zhu, J. et al. Nanofabrication of $30 \mathrm{~nm}$ Au zone plates by e-beam lithography and pulse voltage electroplating for soft X-ray imaging. Microelectron. Eng. 225, 111254 (2020).

34. Arroyo-Hernández, M., Tamayo, J. \& Costa-Krämer, J. L. Stress and DNA assembly differences on cantilevers gold coated by resistive and E-beam evaporation techniques. Langmuir 25, 10633-10638 (2009).

35. Wang, T., Hu, X. \& Dong, S. A renewable SERS substrate prepared by cyclic depositing and stripping of silver shells on gold nanoparticle microtubes. Small 4, 781-786 (2008).

36. Fan, H. et al. Preparation of kapok-polyacrylonitrile core-shell composite microtube and its application as gold nanoparticles carrier. Appl. Surf. Sci. 258, 2876-2882 (2012).

37. Claridge, S. A. et al. Directed assembly of discrete gold nanoparticle groupings using branched DNA scaffolds. Chem. Mater. 17, $1628-1635$ (2005).

38. Young, T.-H. \& Chen, L.-W. Pore formation mechanism of membranes from phase inversion process. Desalination 103, 233-247 (1995).

39. Kim, I.-C., Lee, K.-H. \& Tak, T.-M. Preparation and characterization of integrally skinned uncharged polyetherimide asymmetric nanofiltration membrane. J. Membr. Sci. 183, 235-247 (2001).

40. Wang, Y., Goh, S. H., Chung, T. S. \& Na, P. Polyamide-imide/polyetherimide dual-layer hollow fiber membranes for pervaporation dehydration of C1-C4 alcohols. J. Membr. Sci. 1, 222-233 (2009).

41. Xu, Z.-L., Chung, T.-S. \& Huang, Y. Effect of polyvinylpyrrolidone molecular weights on morphology, oil/water separation, mechanical and thermal properties of polyetherimide/polyvinylpyrrolidone hollow fiber membranes. J. Appl. Polym. Sci. 74, 2220-2233 (1999).

42. Kim, I.-C., Yoon, H.-G. \& Lee, K.-H. Formation of integrally skinned asymmetric polyetherimide nanofiltration membranes by phase inversion process. J. Appl. Polym. Sci. 84, 1300-1307 (2002).

43. Belana, J. et al. Physical ageing studies in polyetherimide ULTEM 1000. Polym. Int. 46, 29-32 (1998). 


\section{Acknowledgements}

This work was supported by the Technology Innovation Program (or Industrial Strategic Technology Development Program-Material Components Technology Development Program) (20011433, Extremely cold-resistant anti-vibration elastomer with EPDM) funded by the Ministry of Trade, Industry and Energy (MOTIE, Korea). This research was supported by Korea Basic Science Institute (National research Facilities and Equipment Center) Grant funded by the Ministry of Education (2019R1A6C1010013).

\section{Author contributions}

S.K. prepared figures and K.-S.J. supervised the whole work in the manuscript. All authors reviewed the manuscript.

\section{Competing interests}

The authors declare no competing interests.

\section{Additional information}

Supplementary information is available for this paper at https://doi.org/10.1038/s41598-020-73506-1.

Correspondence and requests for materials should be addressed to K.-S.J.

Reprints and permissions information is available at www.nature.com/reprints.

Publisher's note Springer Nature remains neutral with regard to jurisdictional claims in published maps and institutional affiliations.

(c) (i) Open Access This article is licensed under a Creative Commons Attribution 4.0 International License, which permits use, sharing, adaptation, distribution and reproduction in any medium or format, as long as you give appropriate credit to the original author(s) and the source, provide a link to the Creative Commons licence, and indicate if changes were made. The images or other third party material in this article are included in the article's Creative Commons licence, unless indicated otherwise in a credit line to the material. If material is not included in the article's Creative Commons licence and your intended use is not permitted by statutory regulation or exceeds the permitted use, you will need to obtain permission directly from the copyright holder. To view a copy of this licence, visit http://creativecommons.org/licenses/by/4.0/.

(C) The Author(s) 2020 